\title{
Tumor-associated macrophages recruited by periostin in intrahepatic cholangiocarcinoma stem cells
}

\author{
JIEHONG ZENG ${ }^{1,2^{*}}$, ZHENGKAI LIU $^{1 *}$, SHUWEN SUN $^{3}$, JIANHONG XIE $^{2}$, LI CAO $^{2}$, \\ PIN LV ${ }^{1}$, SHENGDAN NIE $^{4}$, BAO ZHANG $^{1}$, BOWEN XIE ${ }^{1}$, SIYUAN PENG $^{1}$ and BO JIANG ${ }^{1}$ \\ ${ }^{1}$ Department of Hepatobiliary Surgery, The First Affiliated Hospital of Hunan Normal University-Hunan Provincial People's \\ Hospital, Changsha, Hunan 410006; ${ }^{2}$ Department of General Surgery, Yiyang Central Hospital, Yiyang, Hunan 413000; \\ ${ }^{3}$ Department of Pharmacy, Yiyang Central Hospital, Yiyang, Hunan 413000; ${ }^{4}$ Intistute of Clinical Medical Research, The First \\ Affiliated Hospital of Hunan Normal University-Hunan Provincial People's Hospital, Changsha, Hunan 410006, P.R. China
}

Received August 19, 2016; Accepted November 3, 2017

DOI: $10.3892 / \mathrm{ol} .2018 .8372$

\begin{abstract}
Periostin (POSTN) secreted by intrahepatic cholangiocarcinoma stem cells (ICSCs) serves important roles in promoting tumor progression. The present study aimed to investigate POSTN-recruited tumor-associated macrophages (TAMs) in intrahepatic cholangiocarcinoma (ICC). A total of 50 cases were used to investigate the distribution of ICSCs and TAMs in ICC. HCCC-9810 cells were sorted by cluster of differentiation (CD)44, the expression of POSTN of $\mathrm{CD}_{4} 4^{+}$(cancer stem cells) and CD44 cells (non-cancer stem cells), and medium were evaluated by western blot analysis. HCCC-9810 cells and THP-1 macrophages were used to detect the effects of POSTN on recruiting TAMs in vitro. The present study revealed that $\mathrm{CD} 44^{+}$cells in ICC tissues and the HCCC-9810 cell line were associated with high POSTN secretion levels. Furthermore, POSTN was associated with TAM density in primary ICC tissues. Additionally, POSTN increased the migration of TAMs derived from THP-1 cells. These findings suggested that POSTN secreted by ICSCs may serve important functions in TAM recruitment, and it may be a potential curative strategy to target the tumor microenvironment in ICC.
\end{abstract}

\section{Introduction}

Intrahepatic cholangiocarcinoma (ICC) is the second most common type of malignant primary hepatic tumor

Correspondence to: Dr Bo Jiang, Department of Hepatobiliary Surgery, The First Affiliated Hospital of Hunan Normal University-Hunan Provincial People's Hospital, 61 Jiefang Road, Changsha, Hunan 410006, P.R. China

E-mail: jiangbo_hph@163.com

*Contributed equally

Key words: intrahepatic cholangiocarcinoma, tumor-associated macrophages, periostin, migration, cancer stem cells in numerous areas of the world, including North America, Europe, Australia, and Japan (1). ICC is a type of malignant tumor that originates from peripheral intrahepatic biliary epithelia $(2,3)$. The incidence of ICC and the ICC-associated mortality rate has increased in several regions around the world since the 1970s (4-6). Cancer stem cells (CSCs) are defined as a small subgroup of cancer cells with the ability of self-renewal that may lead to tumor recurrence. A number of studies have suggested that CSCs lead to poor prognosis by promoting tumor recurrence and metastasis (7-9). It has previously been reported that the maintenance of CSC characteristics depends on the tumor microenvironment (TME) (10). The TME consists of tumor cells and stromal cells, including mesenchymal cells, endothelial cells and immune cells, and serves an important role in regulating tumorigenesis, cell invasion and metastasis (11).

Macrophages, a main component of tumor-infiltrating immunocytes, infiltrate a variety of cytokines, chemokines, growth factors and matrix metalloproteases, and contribute to tumor progression and recurrence $(12,13)$. Macrophages are classified into M1 and M2 subtypes due to their polarization manners (14). The M1 subtype appears to be tumor suppressive, whereas the M2 subtype is tumor supportive in tumors (15). M2 subtype macrophages upregulate cluster of differentiation (CD)206, tumor growth factor- $\beta$ and interleukin-10 (16). Macrophages that invade the TME are tumor-associated macrophages (TAMs), expressing similar molecular and functional characteristic of the M2 subtype (12). Abundant macrophage infiltration is a histological feature of ICC, and those macrophages in ICC express similar functional characteristics to the M2 subtype; furthermore, the increased density of macrophages in ICC was associated with a poor prognosis (17). Certain studies have demonstrated that a high density of TAMs was associated with poor prognosis in numerous other types of cancer $(18,19)$. Therefore, researching the molecular mechanisms underlying TAM recruitment may promote the development of therapeutics to effectively improve ICC treatment.

Periostin (POSTN), also known as OSF-2, is a member of the fasciclin family and is a disulfide-linked cell adhesion protein (20). POSTN participates in the multifarious field 
of tumorigenic processes via signaling pathways, including protein kinase $\mathrm{B} /$ phosphoinositide-3 kinase, integrin and Wnt-1 $(21,22)$. Zhou et al $(23)$, revealed that POSTN secreted by stem cells may serve as a chemoattractant for recruiting M2 TAMs in clinical specimens and in an animal model of glioblastoma. In addition, POSTN acts as an important promoter in tumor progression, including growth, angiogenesis, metastasis and invasion, in certain types of malignant cancer $(22,24,25)$. The present study demonstrated that CD $44^{+}$ICC stem cells secrete POSTN, and the density of CD206 ${ }^{+}$TAMs was associated with the expression level of POSTN in ICC.

\section{Materials and methods}

Patients and specimens. A total of 50 patients (age, 43-75 years; median age, 59.8 years; 32 males and 18 females) with curative liver resection and pathology-proven ICC at the Hunan Provincial People's Hospital (Changsha, China) between May 2001 and February 2007 were included in the current study. Tumor stage was re-examined according to the 2009 International Union Against Cancer TNM Classification system (26). The present study was approved by the Hunan Provincial People's Hospital Research Ethics Committee. Written informed consent was obtained from all patients prior to enrollment in the present study.

Immunohistochemical examination. For immunohistochemical analysis of POSTN (TA804575; 1:100; OriGene Technologies, Inc., Beijing, China) and CD206 (SC-376232; 1:100; Santa Cruz Biotechnology, Inc., Dallas, TX, USA), tissue sections (thickness $=4 \mathrm{~mm}$ ) were deparaffinized in $100 \%$ xylene and rehydrated in graded concentrations $(100$, 95, 70 and $50 \%$ ) of ethanol. Following incubation with $1 \%$ bovine serum albumin (BSA; Beijing Dingguo Changsheng Biotechnology Co., Ltd., Beijing, China) in PBS (pH 7.4) at $37^{\circ} \mathrm{C}$ for $30 \mathrm{~min}$, the tissue sections were then incubated with primary antibody for $1 \mathrm{~h}$ at room temperature, followed by incubation with the secondary biotinylated mouse antibody (TA130008; 1:100; OriGene Technologies, Inc., Beijing, China) at $37^{\circ} \mathrm{C}$ for $30 \mathrm{~min}$. Following PBS washing, tissue sections were subsequently treated with streptavidin-peroxidase (S5512; Sigma Aldrich; Merck KgaA, Darmstadt, Germany) at $37^{\circ} \mathrm{C}$ for $30 \mathrm{~min}$. Finally, the results were visualized following a 15-min incubation with diaminobenzidine (DAB; Beyotime Institute of Biotechnology, Haimen, China) at room temperature for $5 \mathrm{~min}$. Horseradish peroxidase was detected using 3,3'-diaminobenzidine (Phoenix Biotechnologies, San Antonio, TX, USA) substrate for $5 \mathrm{~min}$, washed with distilled water, and counterstained with Gill's no. 3 hematoxylin (Sigma-Aldrich; Merck KGaA) at room temperature for $15 \mathrm{sec}$ and mounted. The results were observed under a light microscope (IX51; Olympus Corporation, Tokyo, Japan; magnification, $\mathrm{x} 100)$.

Immunofluorescent staining. For immunohistochemical analysis of POSTN (TA500070; 1:100; OriGene Technologies, Inc.), CD44 (ab51037; 1:100; Abcam, Cambridge, UK) and CD206 (SC-34577; 1:100; Santa Cruz Biotechnology, Inc.), tissue sections were prepared as aforementioned. The sections were then incubated with primary antibody for $1 \mathrm{~h}$ at room temperature. The secondary biotinylated [goat anti-mouse IgG (ab150117; 1:100; Abcam), donkey anti-goat IgG (ab150079; 1:100; Abcam), donkey anti-mouse IgG (ab150131; 1:100; Abcam), Goat anti-mouse IgG (ab150129; 1:100; Abcam)] antibody was applied for identifying primary antibody and incubated at $37^{\circ} \mathrm{C}$ for $30 \mathrm{~min}$. The nuclei were counterstained at room temperature for $10 \mathrm{~min}$ with 4',6-diamidino-2-phenylindole (Sigma-Aldrich; Merck KGaA). The results were observed and representative images were captured using an inverted fluorescent microscope (BX41, Olympus Corporation, Tokyo, Japan) (magnification, x100 and x200).

Cell cultures and cell sorting. HCCC-9810 and THP-1 cells from the Cell Bank of Shanghai Institute of Biological Sciences (Shanghai, China) were cultured in RPMI-1640 medium (Gibco; Thermo Fisher Scientific, Inc., Waltham, MA, USA) supplemented with $10 \%$ fetal bovine serum (FBS; GE Healthcare, Chicago, IL, USA), $100 \mathrm{U} / \mathrm{ml}$ penicillin and $100 \mu \mathrm{g} / \mathrm{ml}$ streptomycin. Cells were incubated in stem cell medium with B27 (Gibco, Thermo Fisher Scientific, Inc.), $10 \mathrm{ng} / \mathrm{ml}$ epidermal growth factor (Prospec-Tany TechnoGene, Ltd., East Brunswick, NJ, USA) and basic fibroblast growth factor (Prospec-Tany TechnoGene, Ltd.) supplement at $37^{\circ} \mathrm{C}$ for $12 \mathrm{~h}$ to expose surface markers, and samples were sorted using a BD FACSVantage SE (BD Biosciences, Franklin Lakes, NJ, USA). Phycoerythrin (PE)-conjugated anti-human CD44 antibody (130-095-194; 1:10; Miltenyi Biotec, Cologne, Germany) was used to label HCCC-9810 cells according to the manufacturer's instructions. CD $44^{+}$and CD44- cell subpopulations were sorted by fluorescence-activated cell sorting with anti-REA (130-104-693; Miltenyi Biotec, Cologne, Germany). The purity of sorted cells was evaluated using a FACSCalibur ${ }^{\mathrm{TM}}$ flow cytometry system (BD Biosciences) and analyzed using flow cytometry on the MACSQuant Analyzer 10. CD44 ${ }^{+}$cells were enriched with stem cell medium and sorted as stem cells; similarly, CD44 cells were used as non-stem cells. All sorted cells were cultured in RPMI-1640 supplemented with $15 \% \mathrm{FBS}$ at $37^{\circ} \mathrm{C}$ for a week. THP-1 cells were treated with phorbol-12-myristate-13-acetate at $100 \mathrm{ng} / \mathrm{ml}$ for $48 \mathrm{~h}$ to generate macrophages.

Western blot analysis. HCCC-9810 cells were lysed using lysis buffer containing $1 \mathrm{mM}$ phenylmethanesulfonyl-fluoride (ST506; Beyotime, Shanghai, China) on ice for $5 \mathrm{~min}$, then total protein of HCCC-9810 and supernatant content was evaluated using a bicinchoninic acid quantitative kit. Quantified protein lysates (5 $\mu \mathrm{g}$ per lane) were resolved using SDS-PAGE (7.5\% gels), transferred onto a polyvinylidene difluoride membrane (EMD Millipore, Billerica, MA, USA). The membrane was blocked with $5 \%$ non-fat milk in TBS with Tween 20 for $30 \mathrm{~min}$ at $25^{\circ} \mathrm{C}$ and immunoblotted with primary antibodies against POSTN (TA500070; 1:500; OriGene Technologies, Inc., Beijing, China) at $4^{\circ} \mathrm{C}$ overnight. Following this, the membrane was incubated with horseradish peroxidase-conjugated secondary antibody (ab6789; 1:4,000; Abcam) at $4^{\circ} \mathrm{C}$ overnight. The blots were visualized using an enhanced chemiluminescence kit (Vazyme, Piscataway, NJ, USA). $\beta$-actin (ab13822; 1:1,000; Abcam) was used as a loading control at $4^{\circ} \mathrm{C}$ overnight. Protein was visualized using FluorChem FC3 (ProteinSimple, San Jose, CA, USA) and ImageJ software (version 1.51p; National Institutes of Health, Bethesda, MD, USA) quantified the band density. 


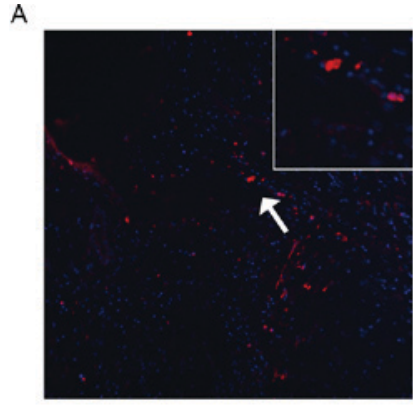

B

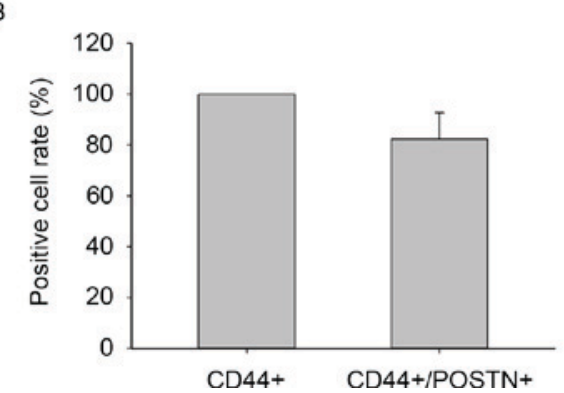

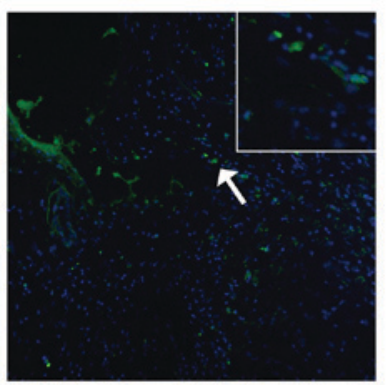

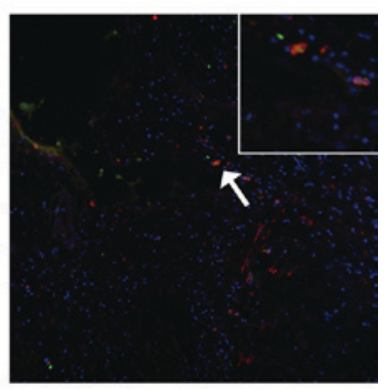

C

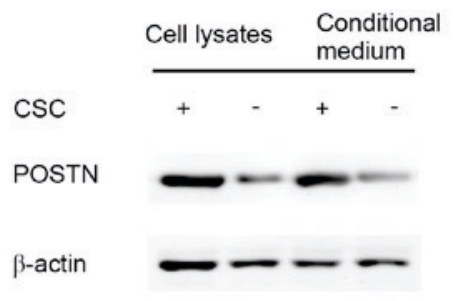

Figure 1. POSTN is secreted by ICSCs in ICC tissues and HCCC-9810 cells. (A) Expression levels of ICSC marker CD44 (red), POSTN (green) and merged (yellow), were observed by immunofluorescent staining in human primary ICC tissues (magnification, x100) and magnified area (arrow). (B) Graphical analysis of (A) identifying that $>80 \%$ of cells were co-stained with CD44 and POSTN. (C) Western blot analysis of POSTN expression in cells and CM. Compared with non-stem cancer cells, CSC lysates and medium revealed POSTN overexpression. POSTN, periostin; ICSC, intrahepatic cholangiocarcinoma stem cells; ICC, intrahepatic cholangiocarcinoma; CD, cluster of differentiation; CSC, cancer stem cell.

Cell migration assays. Transwell chamber assays were used to compare the migratory ability of THP-1-derived macrophages using conditional medium (CM) [NSCCs CM, ICSCs CM, ICSCs CM with POSTN-neutralizing antibody ( $\alpha$-POSTN) and IgG, NSCCs CM with recombinant POSTN (rPOSTN)]. Briefly, phorbol-12-myristate-13-acetate (PMA) treated THP-1-derived macrophages were resuspended in serum-free RPMI-1640 $\left(5 \times 10^{4}\right.$ cells/200 $\left.\mu 1\right)$. BSA (2\%)-RPMI-1640 (500 $\mu 1)$ was added to the upper chambers as the control. Conditioned medium [CM; NSCCs CM, ICSCs CM, ICSCs CM with $\alpha$-POSTN $(10 \mu \mathrm{g} / \mathrm{ml}$; cat no. TA600528; OriGene Technologies, Inc., Beijing, China) or IgG and NSCCs CM with rPOSTN $(0.2 \mu \mathrm{g} / \mathrm{ml})]$ with $10 \%$ FBS was added to the lower chambers. Following a 24-h incubation at $37^{\circ} \mathrm{C}$, the migratory cells to the lower surface of the membrane were fixed with $4 \%$ paraformaldehyde at room temperature for $5 \mathrm{~min}$, stained with Wright Giemsa for $20 \mathrm{~min}$ at room temperature, and counted and imaged using a microscope (BX41; Olympus Corporation, Tokyo, Japan; magnification, $\mathrm{x} 400)$.

Statistical analysis. Data were presented as the mean \pm standard error of the mean from at least three samples or experiments per data point. Differences between the groups were analyzed by one-way analysis of variance with Fisher's Least Significant Difference test as a post-hoc using SPSS version 15.0 (SPSS, Inc., Chicago, IL, USA). $\mathrm{P}<0.05$ was considered to indicate a statistically significant difference.

\section{Results}

POSTN is secreted by ICSCs in ICC tissues and HCCC-9810 cells. To investigate the latency association between POSTN expression and distribution of CSCs in cholangiocarcinoma, the present study determined the expression levels of POSTN and CSC marker CD44 in human primary ICC samples by evaluating immunofluorescence. The findings revealed that POSTN is preferentially expressed by $\mathrm{CD}_{4} 4^{+}$cancer cells and located in the area around ICSCs (Fig. 1A and B). To determine the differential expression of POSTN between ICSCs and non-stem cancer cells (NSCCs), the present study examined the expression levels of POSTN in ICSCs and NSCCs of the HCCC-9810 cell line by western blot analysis (Fig. 1C). The results demonstrated that ICSCs expressed higher levels of POSTN compared with NSCCs. Furthermore, ICSC conditioned medium also contained higher POSTN protein levels compared with matched NSCC conditioned medium (Fig. 1C). These results suggest that POSTN is preferentially secreted by ICSCs.

POSTN is associated with TAM density in primary ICC. The association between POSTN expression level and TAM density was first evaluated by immunofluorescence. The results demonstrated that TAM-labeled marker (CD206) was accumulated in a POSTN-abundant location (Fig. 2A). Immunohistochemistry demonstrated that high levels of POSTN and high levels of TAM markers were identified in ICC (Fig. 2B and C). These results suggest that POSTN expression levels had a positive association with the number of TAMs in ICC.

POSTN promotes migratory ability of TAMs derived from human macrophage-like THP-1 cells. To clarify the mechanism underlying POSTN action as an effective ICSC-secreted chemotaxin, migration of PMA-primed macrophage-like THP-1 cells were evaluated by Transwell assays. Conditioned 

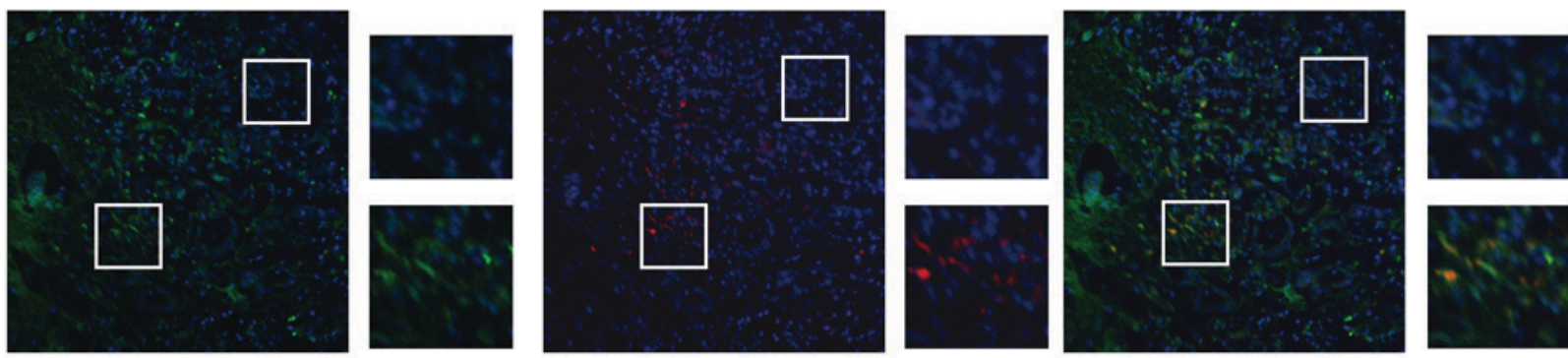

POSTN $^{H}$

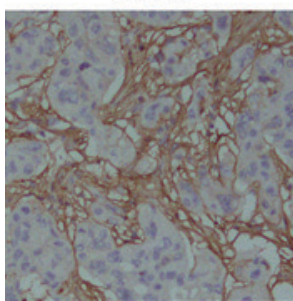

$\mathrm{CD} 206^{\mathrm{H}}$

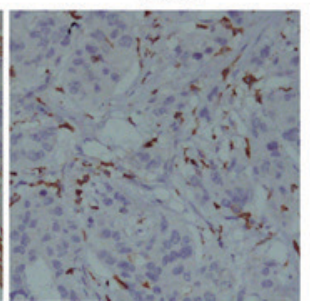

POSTN $^{\mathrm{L}}$

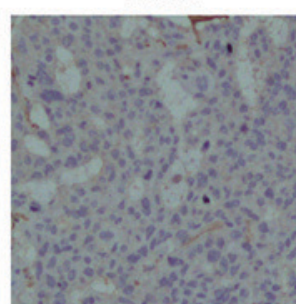

CD206 ${ }^{\mathrm{L}}$

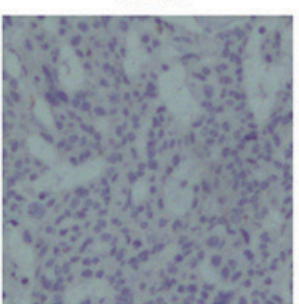

Figure 2. POSTN is associated with TAM density in primary ICC. (A) Representative immunofluorescence images showing POSTN (green) and TAM marker CD206 (red) expression in ICC tissues (magnification, x200) and selected areas (magnification, x200). (B) Representative immunohistochemical images showing POSTN and CD206 staining (magnification, x200). (C) A total of 54\% of ICC cases presented POSTN ${ }^{\mathrm{H}}$ and CD206 ${ }^{\mathrm{H}}$ staining, and $26 \%$ of ICC cases presented POSTN ${ }^{\mathrm{L}}$ and CD206 ${ }^{\mathrm{L}}$ staining; however, $12 \%$ of ICC cases presented POSTN ${ }^{\mathrm{H}}$ and CD206 ${ }^{\mathrm{L}}$ staining, and $8 \%$ of ICC cases presented POSTN ${ }^{\mathrm{L}}$ and CD206 ${ }^{\mathrm{H}}$ staining. The majority (80\%) of ICC cases revealed that POSTN expression was positively associated with TAM density. POSTN, periostin; TAM, tumor-associated macrophage; ICC, intrahepatic cholangiocarcinoma; CD, cluster of differentiation; H, high level; L, low level.

A
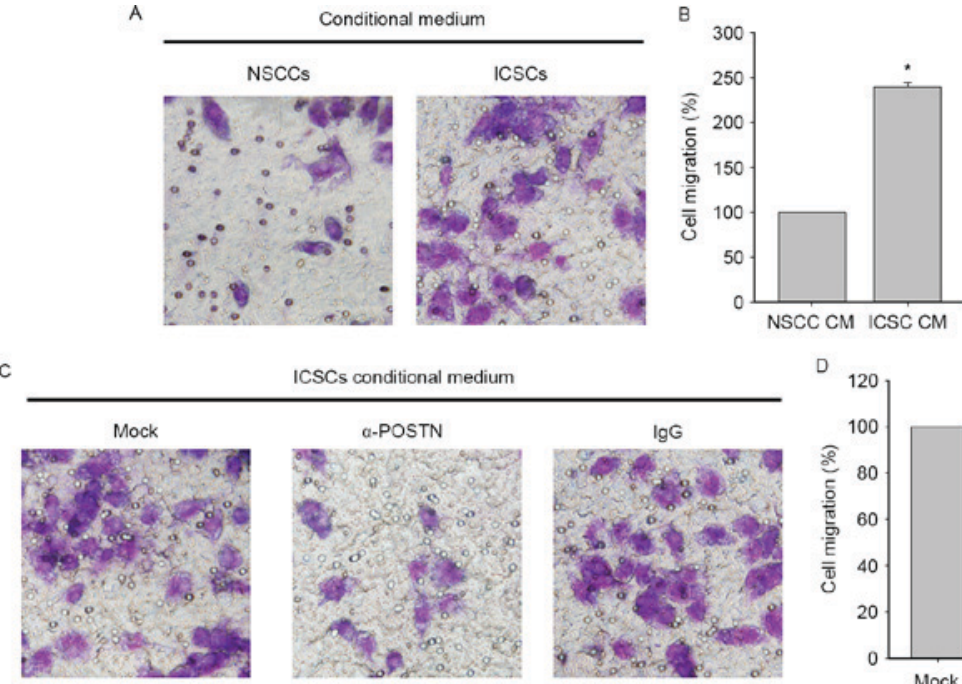

ICSCs conditional medium
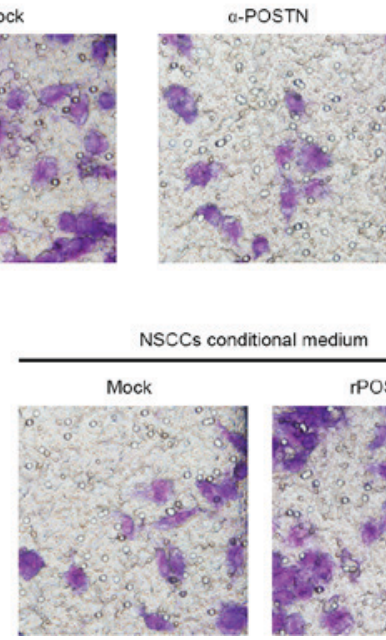

NSCCs conditional medium
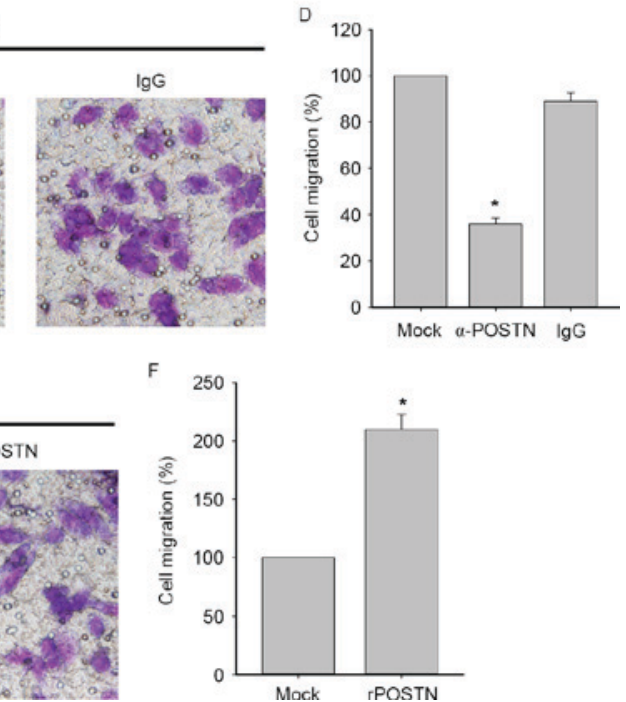

Figure 3. POSTN promotes the migration of TAMs. (A) Transwell assay showing comparison of TAMs migration toward CM from NSCCs and ICSCs in HCCC-9810 cells (magnification, $\mathrm{x} 400$ ) and (B) the graphical analysis. Data are presented as mean $\pm \mathrm{SD}(\mathrm{n}=3)$. $\mathrm{P}<0.05$, migrated TAMs towards ICSC CM vs. NSCC CM. The analysis identified that the increased TAMs cell migration toward ISCCs CM relative to NCSCs CM. (C) Comparison of TAMs migration toward ICSC CM or following treatment with anti-POSTN $(10 \mu \mathrm{g} / \mathrm{ml})$ antibody or IgG (magnification, $\mathrm{x} 400)$ and (D) its graphical analysis. Data are presented as mean $\pm \mathrm{SD}(\mathrm{n}=3)$. ${ }^{*} \mathrm{P}<0.05$, migrated TAMs towards to $\alpha$-POSTN ICSC CM vs. ICSC CM and IgG ICSC CM. (E) Comparison of invading TAMs toward NSCC CM or following treatment with $\mathrm{rPOSTN}(0.2 \mu \mathrm{g} / \mathrm{ml})$ or IgG (magnification, $\mathrm{x} 400)$ and the (F) graphical analysis. Data are presented as mean \pm SD $(\mathrm{n}=3)$. ${ }^{*} \mathrm{P}<0.05$, migrated TAMs towards to rPOSTN NSCC CM vs. NSCC CM. POSTN, periostin; TAM, tumor-associated macrophage; NSCCs, non-stem cancer cells; ICSCs, intrahepatic cholangiocarcinoma stem cells; IgG, immunoglobulin G; r, recombinant; CM, conditional medium; SD, standard deviation; Ig, immunoglobulin. 
medium from ICSCs attracted significantly more TAMs than the medium from matched NSCCs (Fig. 3A and B). Subsequently, the present study used $\alpha$-POSTN to deplete POSTN expression. As presented in Fig. 3C and D, the depletion of POSTN in ICSC-CM suppressed the promoting effect of macrophage migration in vitro. The capacity of POSTN to increase invasiveness of human monocytes was also demonstrated in the present study (Fig. 3E and F). Collectively, these results demonstrate that POSTN preferentially secreted by ICSCs had an effective capacity to attract macrophages.

\section{Discussion}

The present study observed a large level of CD206 ${ }^{+}$macrophage infiltration in parts of the ICC tumor niche. TAMs in cancerous tissues are regarded as immunosuppressive cells that have a tumor supportive role (27). Therefore, investigating the molecular mechanisms underlying TAM recruitment may contribute to improvement of ICC treatment.

It has been reported that tumors recruit TAMs by secreting the $\mathrm{CC}$ chemokine ligand 2 and soluble colony-stimulating factor 1 in tumors (28-30). The present study revealed that TAMs were concentrated in POSTN-abundant regions in ICCs. Similarly, immunohistochemistry analysis demonstrated the following: In ICC, tumor tissues with higher expression levels of POSTN contained higher densities of TAMs, revealing a positive association between POSTN levels and TAM density in human ICCs. The present study also revealed that POSTN was secreted by ICSCs. In order to determine the differential expression of POSTN in CD44+ ICSCs, the present study observed the expression of POSTN in matched ICSCs and NSCCs. These results demonstrated that ICSCs preferentially expressed markedly higher POSTN levels compared with NSCCs. Consistently, CM from ICSCs contained higher levels of POSTN protein compared with that from matched NSCCs. These results indicated that POSTN was preferentially produced by ICSCs rather than NSCCs.

To further elucidate whether ICSCs secreting POSTN had potent capacity to recruit TAMs, cell migration assays were performed in vitro. The Transwell assay identified that TAMs of the CM group had higher migratory ability compared with the NSCCs group. The present study also revealed the migratory ability of the CM group with anti-POSTN antibody exhibited a decreased migratory ability. Subsequently, the present study demonstrated that the migratory ability of NSCCs was increased by rPOSTN. These results revealed that POSTN preferentially secreted by ICSCs displays potent ability to attract TAMs.

Trabectedin has demonstrated antitumor activity by targeting TAMs (31). The present study revealed that the underlying mechanisms of TAM recruitment by ICSC-secreted POSTN may be responsible for the crosstalk of TAMs and ICSCs. In addition, therapeutic targeting of the immune TME may synergize with current immunotherapies to effectively increase survival of ICC patients.

\section{Acknowledgements}

The present study was supported by the Project of the National Natural Science Foundation of China (grant no. 81001107), the
Project of the Scientific Research Fund of Hunan Provincial Education Department (grant no. 15A114) and the Project of Scientific Research Fund of Hunan Science and Technology Education Department (grant no. 2015SK2050).

\section{References}

1. Goodman ZD: Neoplasms of the liver. Mod Pathol 20: S49-S60, 2007.

2. Nakanuma Y, Harada K, Ishikawa A, Zen Y and Sasaki M: Anatomic and molecular pathology of intrahepatic cholangiocarcinoma. J Hepatobiliary Pancreat Surg 10: 265-281, 2003.

3. Parkin DM, Bray F, Ferlay J and Pisani P: Global cancer statistics, 2002. CA Cancer J Clin 55: 74-108, 2005.

4. Okuno M,Ebata T, Yokoyama Y, Igami T, Sugawara G, Mizuno T, Yamaguchi $\mathrm{J}$ and Nagino M: Appraisal of inflammation-based prognostic scores in patients with unresectable perihilar cholangiocarcinoma. J Hepatobiliary Pancreat Sci 23: 636-642, 2016.

5. Shaib Y and El-Serag HB: The epidemiology of cholangiocarcinoma. Semin Liver Dis 24: 115-125, 2004.

6. Patel T: Worldwide trends in mortality from biliary tract malignancies. BMC Cancer 2: 10, 2002.

7. Sun YF, Xu Y, Yang XR, Guo W, Zhang X, Qiu SJ, Shi RY, Hu B, Zhou J and Fan J: Circulating stem cell-like epithelial cell adhesion molecule-positive tumor cells indicate poor prognosis of hepatocellular carcinoma after curative resection. Hepatology 57: 1458-1468, 2013

8. Chiba T, Zheng YW, Kita K, Yokosuka O, Saisho H, Onodera M, Miyoshi H, Nakano M, Zen Y, Nakanuma Y, et al: Enhanced self-renewal capability in hepatic stem/progenitor cells drives cancer initiation. Gastroenterology 133: 937-950, 2007.

9. Wu XZ and Yu XH: Bone marrow cells: The source of hepatocellular carcinoma? Med Hypotheses 69: 36-42, 2007.

10. Borovski T, De Sousa E Melo F, Vermeulen L and Medema JP: Cancer stem cell niche: The place to be. Cancer Res 71: 634-639, 2011.

11. Schiavoni G, Gabriele L and Mattei F: The tumor microenvironment: A pitch for multiple players. Front Oncol 3: 90, 2013.

12. Biswas SK, Allavena P and Mantovani A: Tumor-associated macrophages: Functional diversity, clinical significance, and open questions. Semin Immunopathol 35: 585-600, 2013.

13. Cook J and Hagemann T: Tumour-associated macrophages and cancer. Curr Opin Pharmacol 13: 595-601, 2013.

14. Sielska M, Przanowski P, Wylot B, Gabrusiewicz K, Maleszewska M, Kijewska M, Zawadzka M, Kucharska J, Vinnakota K, Kettenmann H, et al: Distinct roles of CSF family cytokines in macrophage infiltration and activation in glioma progression and injury response. J Pathol 230: 310-321, 2013.

15. Staudt ND, Jo M, Hu J, Bristow JM, Pizzo DP, Gaultier A, VandenBerg SR and Gonias SL: Myeloid cell receptor LRP1/CD91 regulates monocyte recruitment and angiogenesis in tumors. Cancer Res 73: 3902-3912, 2013.

16. Zhang G, Guo L, Yang C, Liu Y, He Y, Du Y, Wang W and Gao F: A novel role of breast cancer-derived hyaluronan on inducement of M2-like tumor-associated macrophages formation. Oncoimmunology 5: e1172154, 2016.

17. Oishi K, Sakaguchi T, Baba S, Suzuki S and Konno H: Macrophage density and macrophage colony-stimulating factor expression predict the postoperative prognosis in patients with intrahepatic cholangiocarcinoma. Surg Today 45: 715-722, 2015.

18. Mantovani A and Sica A: Macrophages, innate immunity and cancer: Balance, tolerance, and diversity. Curr Opin Immunol 22: 231-237, 2010.

19. Liu JY, Yang XJ, Geng XF, Huang CQ, Yu Y and Li Y: Prognostic significance of tumor-associated macrophages density in gastric cancer: A systemic review and meta-analysis. Minerva Med 107: 314-321, 2016.

20. Bao S, Ouyang G, Bai X, Huang Z, Ma C, Liu M, Shao R, Anderson RM, Rich JN and Wang XF: Periostin potently promotes metastatic growth of colon cancer by augmenting cell survival via the Akt/PKB pathway. Cancer Cell 5: 329-339, 2004.

21. Baril P, Gangeswaran R, Mahon PC, Caulee K, Kocher HM, Harada T, Zhu M, Kalthoff H, Crnogorac-Jurcevic T and Lemoine NR: Periostin promotes invasiveness and resistance of pancreatic cancer cells to hypoxia-induced cell death: Role of the beta4 integrin and the PI3k pathway. Oncogene 26: 2082-2094, 2007.

22. Malanchi I, Santamaria-Martínez A, Susanto E, Peng H, Lehr HA, Delaloye JF and Huelsken J: Interactions between cancer stem cells and their niche govern metastatic colonization. Nature 481: 85-89, 2011. 
23. Zhou W, Ke SQ, Huang Z, Flavahan W, Fang X, Paul J, Wu L, Sloan AE, McLendon RE, Li X, et al: Periostin secreted by glioblastoma stem cells recruits M2 tumour-associated macrophages and promotes malignant growth. Nat Cell Biol 17: 170-182, 2015.

24. Michaylira CZ, Wong GS, Miller CG, Gutierrez CM, Nakagawa $H$, Hammond R, Klein-Szanto AJ, Lee JS, Kim SB, Herlyn M, et al: Periostin, a cell adhesion molecule, facilitates invasion in the tumor microenvironment and annotates a novel tumor-invasive signature in esophageal cancer. Cancer Res 70: 5281-5292, 2010.

25. Liu Y, Li F, Gao F, Xing L, Qin P, Liang X, Zhang J, Qiao X, Lin L, Zhao $\mathrm{Q}$ and Du L: Periostin promotes tumor angiogenesis in pancreatic cancer via Erk/VEGF signaling. Oncotarget 7: 40148-40159, 2016

26. Nathan H, Aloia TA, Vauthey JN, Abdalla EK, Zhu AX, Schulick RD, Choti MA and Pawlik TM: A proposed staging system for intrahepatic cholangiocarcinoma. Ann Surg Oncol 16: 14-22, 2009.

27. Mantovani A, Sica A, Sozzani S, Allavena P, Vecchi A and Locati M: The chemokine system in diverse forms of macrophage activation and polarization. Trends Immunol 25: 677-686, 2004.
28. Popivanova BK, Kostadinova FI, Furuichi K, Shamekh MM, Kondo T, Wada T, Egashira K and Mukaida N: Blockade of a chemokine, CCL2, reduces chronic colitis-associated carcinogenesis in mice. Cancer Res 69: 7884-7892, 2009.

29. Qian BZ, Li J, Zhang H, Kitamura T, Zhang J, Campion LR, Kaiser EA, Snyder LA and Pollard JW: CCL2 recruits inflammatory monocytes to facilitate breast-tumour metastasis. Nature 475: 222-225, 2011.

30. Pyonteck SM, Gadea BB, Wang HW, Gocheva V, Hunter KE, Tang LH and Joyce JA: Deficiency of the macrophage growth factor CSF-1 disrupts pancreatic neuroendocrine tumor development. Oncogene 31: 1459-1467, 2012.

31. Germano G, Frapolli R, Belgiovine C, Anselmo A, Pesce S, Liguori M, Erba E, Uboldi S, Zucchetti M, Pasqualini F, et al: Role of macrophage targeting in the antitumor activity of trabectedin. Cancer Cell 23: 249-262, 2013. 